\title{
ANÁLISIS DEL CURRÍCULO Y OTROS ACTORES DEL PROCESO EDUCATIVO PARA IDENTIFICAR EL EJE AMBIENTAL: INFORME PROYECTO
}

CURRICULUM AND OTHER ACTORS INVOLVED IN THE EDUCATIONAL PROCESS ANALYSIS CONCERNING THE ENVIRONMENTAL AXIS: PROJECT REP

ANÁLISE DO CURRÍCULO E DE OUTROS ATORES DO PROCESSO EDUCACIONAL PARA IDENTIFICAR O EIXO AMBIENTAL: RELATÓRIO DE PROJETO

\author{
Dr. Ramón Bedolla Solano* \\ rambedsol@hotmail.com, \\ M.C. Adriana Miranda Esteban** \\ M.C. Adriana Mirab_o5@hotmail.com \\ Dr. Juan José Bedolla Solano*** \\ Dr. Benjamin Castillo Elias ${ }^{* * * *}$ \\ bcastilloe@hotmail.com \\ Dr. Oscar Sánchez Adame***** \\ msposcar@hotmail.com \\ Dra. Herlinda Gervacio Jiménez \\ Lindagjo9@hotmail.com
}

REVISTA PEDAGÓGICA

Revista do Programa de Pós-graduação em Educação da Unochapecó | ISSN 1984-1566 Universidade Comunitária da Região de Chapecó | Chapecó-SC, Brasil Como referenciar este artigo: SOLANO, R. B.; ESTEBAN, A. M.; SOLANO, J. J. B.; ELIAS, B. C.; ADAME, O. S.; JIMÉNEZ, H. G. Análisis del currículo y otros actores del proceso educativo para identificar el eje ambiental: informe proyecto. Revista Pedagógica, Chapecó, v. 18, n. 39, p. 16-29, set./dez. 2016. DOI: http://dx.doi.org/10.22196/rp.v18i39.3613

RESUMEN: Referente a la primer etapa de un proyecto de investigación que se tiene registrado en la Universidad Autónoma de Guerrero (UAGro) durante el periodo 2015-2016 y que entre sus objetivos se plantea un proceso para integrar el eje medio ambiente en planes de estudio de licenciatura en la UAGro, se presenta un análisis que se derivó de un diagnóstico realizado de esta etapa. El diagnóstico, comprendió en indagar a través de instrumentos, como rubricas, entrevistas, encuestas a actores del proceso educativo y al currículo en tres programas educativos para identificar la presencia del eje medio ambiente. Es una investigación mixta y la información obtenida a través de los instrumentos fue procesada y analizada cuantitativa y cualitativamente. Los resultados de este análisis arrojaron que el elemento medio ambiente escasamente está contemplado en el currículo de las instituciones investigadas.

Palabras claves: Currículo, dimensión ambiental, Educación Ambiental, transversalidad.

Abstract: This paper contains an analysis which derives from a diagnosis made from the first stage of a research project registered by the Autonomous University of Guerrero (UAGro) during 2015-2016 period. Among its goals arises a process to integrate the environmental axis into UAGro graduation curriculums. For this phase were used instruments such as rubrics, interviews, research concerning actors involved in the educational process within three programs to verify the axis presence, leading to a mixed investigation where the data obtained was analyzed quantitatively and qualitatively. As a conclusion, it is shown that that the environmental element lacks approach in the researched institutions.

Keywords: Curriculum, Environmental Dimension, Environmental Education, Transversality.

RESUMO: Referindo-se à primeira etapa de um projeto de pesquisa que tem registado com a Universidade Autónoma de Guerrero (UAGro) durante o período 20152016 e que entre os seus objetivos surge um processo para integrar o eixo meio ambiente no currículo de licenciatura na UAGro, apresenta-se uma análise que derivou de um diagnóstico feito desta fase. O diagnóstico, indagou através de instrumentos como rubricas, entrevistas, pesquisas a atores envolvidos no processo educativo e ao currículo em três programas educacionais para identificar a presença de eixo ambiental. É uma investigação mista e o conjunto de informações obtidas através dos instrumentos foram processados e analisados quantitativamente e qualitativamente. Os resultados desta análise mostrou que o elemento ambiental é pouco abordado no currículo das instituições pesquisadas.

Palavras-chave: currículo, dimensão ambiental, educação ambiental, transversalidade. 
* Dr. en Ciencias de la Educación y Dr. en Desarrollo Regional, Universidad Autónoma de Guerrero (UAGro), México.Unidad Académica de Sociología -Unidad Académica de Desarrollo Regional (Programa Educativo: Doctorado en Ciencias Ambientales PNPC-CONACYT), Cuerpo Académico: Educación y Sustentabilidad.

** M.C en Ciencias de la Educación, Universidad Autónoma de Guerrero, Mèxico. Cuerpo Académico.

*** Dr. en Desarrollo Regional, Instituto Tecnológico de Acapulco, México. Cuerpo Acadêmico: Educación y Sustentabilidad.

**** Dr. en Ciencias Ambientales, Unidad Académica de Medicina Veterinaria y Zootecnia, Universidad Autônoma de Guerrero, México. Cuerpo Acadêmico: Educaciòn y Sustentabilidad.

***** Dr. en Desarrollo Regional, Facultad de Medicina, Universidad Autónoma de Guerrero, México. Cuerpo Acadêmico: Educación y Sustentabilidad.

\section{INTRODUCCIÓN}

Desde casi un poco más de una década la Universidad Autónoma de Guerrero (UAGro), institución en México, se ha propuestoincorporar el elemento medio ambiente en los planes de estudio, principalmente en los de nivel licenciatura.En el documento (Modelo Educativo y Académico de la UAG 2004),el elemento en cuestión se hace presente en los ejes transversales de formación. Aunado a ello plantea la "transversalidad", como un método para desarrollar temas emergentes como lo es el medio ambiente en el currículo. "La transversalidad como una nueva manera de ver la realidad y vivir las relaciones sociales desde una visión holística o de totalidad”. En este tenor, también se expresa que los contenidos trasversales (temas y/o tópicos transversales) que hacen referencia, preferentemente, a contenidos emergentes e integradores. Entre estos temas se encuentran, derechos humanos, pobreza, medio ambiente, etc. Este documento sufrió una reforma en el año 2013y paso del nombre anterior a "Modelo Educativo: Hacia una educación de calidad con inclusión social", lo cual sigue manteniendo el elemento medio ambiente en los ejes transversales y lo considera como tema transversal al igual que otros, y con ello también manifiesta que, estos temas, Son “...instrumentos globalizantes de carácter interdisciplinario que recorren la totalidad de un currículo; son condiciones favorables para proporcionar a los alumnos una mayor formación en aspectos sociales, ambientales o de salud" [Botero 2008:Modelo Educativo: 2013b]. Estas aseveraciones sugieren que en todos los niveles que imparte la institución en cuestión se incorpore el elemento medio ambiente, con un enfoque transversal para que se coadyuve al desarrollo sustentable. Muchas instituciones que imparten educación en el mundo ya lo han hecho, es decir, ya integran el elemento "ambiente" en planes de estudio, las universidades lo hacen también, sin embargo hay algunas que todavía no, otras lo intentan aun. Un ejemplo, se dio en la especialidad de Ciencias NaturalesFacultad de Educación- Universidad Nacional Federico Villarreal en Perú, donde se analizó el Plan Curricular-2005, encontrándose que solamente, 13 asignaturas de 80; tenían contenidos relacionados con el medio ambiente y que las asignaturas del área de la Especialidad, han influenciado en el desarrollo de las actitudes ambientales: en la dimensión cognitiva; pero, el desarrollo de las actitudes: efectiva y reactiva, prácticamente, eran nulo. Con ello se sugirió que se incorpore al interior de los contenidos temáticos el modelo de la transversalidad ambiental, el cual permitirá desarrollar el proceso de manera interdisciplinaria y mejorando las actitudes ambientales en los estudiantes en beneficio de las futuras generaciones (SALVADOR, S. M. 2013, p. 1). El referente para integrar el ambiente en el currículo, es la "problemática ambiental" que se vive a nivel mundial, y esto no es de ahora. Organizaciones a nivel 
mundial como las Naciones Unidas (ONU) desde hace algunas décadas han desarrollado conferencias, cumbres y otros eventos a nivel mundial para tratar el asunto que aqueja al medio ambiente y una de las estrategias que han propuesto para contrarrestar problemáticas y prevenir el deterioro ambiental ha sido la estrategia de la "Educación Ambiental(EA)" que se puedeimplementar dentro o fuera de una institución educativa. Con relación al campo educativo, la (EA) puede ser incorporada vía currículo. La razón para llevar a cabo este tipo de educación, seria incorporando el elemento "medio ambiente" en un plan de estudio. La (EA) es un proceso, no es una materia, en el momento de desarrollar un tema que se relacione con medio ambiente, desde ese momento, se pone en prácticaEducación Ambiental. La incorporacióndel elemento medio ambiente o bien la (EA) en el campo educativo implica desarrollar un proceso con orientaciones hacia la preservación del medio. Este proceso se ha determinado a través de enfoques para su desarrollo, es decir, "Educación Ambiental" o "Educación Ambiental para la sustentabilidad", el primero se orienta solamente a una educación que tiene como propósito cuidar y preservar los recursos naturales y el segundo, es más amplio, es educar, considerando el medio ambiente o bien la naturaleza, relacionándola con sociedad, aspectos económicos, culturales, entre otros. De ello, se comprende que desde los inicios de la (EA) esta, se orientaba solamente a educar para preservar la naturaleza, más adelante, para cuidar el medio, pero considerando los demás elementos que están dentro del medio. Este último enfoque debe su orientación a los conceptos de "desarrollo" y "sustentabilidad" que fueron tocados en las reuniones organizadas por la ONU que tocaron la temática medio ambiente, como lo fue la reunión de Tibilisi de 1977, después en 1987 en el informe Brutland, la Reunión de Rio en 1992 y posteriormente en la Sudáfrica en el año 2002. Aunque estos conceptos surgieron en los 70 `s, estos se adhirieron a la (EA) por la década de los 90 `s. Es por esta razón que hoy se habla de una Educación Ambiental para el Desarrollo Sostenible (EAS)no obstante (EA), se comprende con dicho término. Actualmente se sugiere que los currículos integren (EA), con enfoque para la sustentabilidad, además, como proceso trasversal y no como una materia u otra disciplina.¿Pero que es la transversalizaciòn?, es un proceso que recorre el currículo con contenidos que están presentes en todo el proceso educativo. Dichos contenidos son culturalmente relevantes y necesarios para la vida y la convivencia, ya que dan respuesta a problemas sociales y contribuyen a formar de manera especial el modelo de ciudadano que demanda la sociedad. Son temas que no necesariamente tienen que conformar una asignatura en particular ni recibir un tratamiento especial dentro del currículo, sino que deben abordarse en todas las áreas que lo integran y en toda situación concreta de aprendizaje (PALOS, 1998: HONDURAS, 2009, p.6). La Educación 
Ambiental, esta, no se trata de una disciplina o materia, sino de una dimensión o enfoque educativo integrador $\mathrm{u}$ holístico, como se le suele llamar algunas veces, que debe permear todo el espacio educativo, sea éste curricular o no (GUIER, 2004). La Educación Ambiental (EA) no es un campo de estudio cono la química, física, biología o ecología. Que (EA) es un proceso y que en realidad el termino sería Educación Para el Desarrollo Sostenible porque es más comprensible y especifico, lo cual evitaría confusiones, ya que indica claramente el propósito del esfuerzo educativo: Educación sobre el Desarrollo Sostenible, el cual es en realidad la meta de la Educación Ambiental (CHAGOLLÀN, et al. 2006, p. 16). Con respecto al diagnóstico realizado en tres programas educativos se constató finalmente que estos no contemplaban la EA como lo sugiere el modelo de la institución, más adelante se describen los resultados detalladamente.

\subsection{Incorporación del elemento medio ambiente como una Educación Ambientalpara coadyuvar en el desarrollo sostenible}

Como se ha mencionado, la incorporación del elemento ambiental en el currículo se debe orientar desde la perspectiva del desarrollo, con enfoque interdisciplinario y transversal, considerando las sugerencias de las reuniones y conferencias que se han desarrollado a nivel mundial. En 1977, la Conferencia Intergubernamental sobre Educación Ambiental organizada por la UNESCO en cooperación con el Programa de las Naciones Unidas para el Medio Ambiente (PNUMA) celebrada en la ciudad de Tbilisi ex República Socialista Soviética de Giorgia, en dicha conferencia se dan las orientaciones para la incorporación de la llamada dimensión ambiental en todo el sistema educativo (informal, formal básico, universitario), desde un enfoque interdisciplinario (ZAMBRANO, et al. 2010, pp. 202-203). Para Novo (1996), una de las consecuencia de la Conferencia de Tbilisi, es el informe de la Comisión Mundial sobre Ambiente y Desarrollo (WCED) presentado en 1987, donde se llegó a la conclusión de que la alternativa a los graves y diversos problemas ambientales que enfrenta el planeta, podrán ser resueltos o minimizados dentro de la teoría del desarrollo sostenible. Dicha propuesta, se convierte en el norte paradigmático de la educación ambiental en las décadas de los 1990 y de 2000 (García, op. cit) [ZAMBRANO, et al. b]. En la declaración, surgida de la Segunda Conferencia Mundial sobre Medio Ambiente o Cumbre de Río, Río de Janeiro 1992, se dice expresamente que la EA es indispensable para la modificación de actitudes y para desarrollar comportamientos compatibles con un desarrollo sostenible, y por ello, debe ser introducida en todos los niveles escolares, reexaminando los programas 
escolares y los métodos de educación. El programa o agenda 21 destaca la vinculación entre ambiente y desarrollo y la importancia crítica de una EA escolar y extraescolar, transversal e interdisciplinaria que abarque todos los ámbitos -económicos, ambientales, sociales, de desarrollo humano- para el desarrollo sostenible (MACEDO, et al. 2007, p.33). Los aportes de la Cumbre Mundial Sobre Desarrollo Sostenible, Johannesburgo, 2002 refuerzan la idea que la educación para la sostenibilidad deberá potenciar el compromiso con valores, principios, actitudes y comportamientos $\mathrm{y}$, más concretamente, con una noción de justicia y equidad ampliamente comprendida, así como, el sentimiento de compartir un destino común con todos los pueblos. Esta educación, por lo tanto, no debe limitarse a enfatizar solamente las disciplinas, áreas o temas que mejoren la comprensión del contexto y la naturaleza (MACEDO, et al. 2007b). El papel de la educación ha sido reconocido hace mucho tiempo por organismos internacionales como la UNESCO. El año 2002 se celebró la Cumbre Mundial sobre el Desarrollo Sostenible de Johannesburgo, oportunidad en que se ratificó los compromisos a favor de un desarrollo sostenible a todos los niveles, desde el local al mundial. De esta manera en la Cumbre se propuso la proclamación del Decenio de las Naciones Unidas de la Educación con miras al desarrollo sostenible, señalando que, la educación y el aprendizaje eran aspectos fundamentales de este último y uno de los objetivos que se propone es proporcionar a los países nuevas oportunidades para incorporar la Educación para el Desarrollo Sostenible (EDS) en sus reformas educativas. Entonces para alcanzar este propósito el decenio propone reorientar los programas educativos actuales y futuras, es importante reconsiderar y revisar el sistema educativo, desde la escuela infantil, hasta la universidad, a fin de que transmita los conocimientos, actitudes, perspectivas y valores relacionados con la sostenibilidad (BRAVO, 2011. p.58).

\subsubsection{La problemática ambiental, tema emergente en el currículo}

Muchos problemas son los que están afectando al medio ambiente y que finalmente impactan de manera negativamente en los demás aspectos sociales. Entre ellos se mencionan, los residuos (basura), la contaminación del agua, deforestación, etc.). En muchos casos el hombre es responsable de estos daños, lo cual, es necesario que modifique sus actitudes y comportamientos.En el caso de los residuos, si bien, la mayor parte de la basura producida en el mundo (estimada en más de 2 billones de toneladas anuales) se entierra o se incinera, las predicciones para las próximas décadas no son muy alentadoras. Algunas 
sociedades sostienen que los rellenos sanitarios no son los adecuados para el destino final de los residuos. Los líquidos lixiviados de la basura (que son cien veces más contaminantes que los líquidos cloacales). Las cuencas de ríos y lagos, se convierten a menudo en reservorios de basura, desagües de minas o depósitos de residuos agrícolas e industriales. Como corolario, la mayor parte de las aguas residuales se vierte directamente en los ríos, lagos o canales sin tratamiento de ningún tipo. En ciertas regiones de Latinoamérica, se han librado batallas por el agua, como por ejemplo en Argentina, donde presenta serios problemas en varios cursos de agua, seriamente contaminados. La desprotección que padece el medio ambiente con una constante agresión por parte del hombre por no haber medido las consecuencias al detenerse en ellas, y no haber atacado y modificado férreamente las causas que llevaron a este presente (MICILIO, 2009, p. 69-74).A medida que se han ido acelerando las presiones de los seres humanos en el sistema Tierra nos hemos acercado a varios umbrales críticos mundiales, regionales y locales. Las consecuencias de los cambios complejos y no lineales en el sistema Tierra ya están teniendo graves consecuencias para el bienestar humano, como son: sequías combinadas con presiones sociales y económicas, que afectan la seguridad humana; aumento de la temperatura media por encima de ciertos umbrales en determinados lugares, lo que ha tenido importantes consecuencias en la salud humana, como un aumento de los casos de malaria; aumento de la frecuencia y la gravedad de fenómenos climáticos, como inundaciones y sequías, a niveles sin precedentes que afectan tanto el capital natural como la seguridad humana; variación cada vez más rápida de la temperatura y aumento del nivel del mar que influyen en el bienestar humano en determinados lugares (PNUMA, 2012, p. 6-7).Los problemas ambientales amenazan la salud, el sustento y vida de las personas y pueden causar guerras y amenazar a las generaciones futuras (HOPWOOD, 2005, p. 39).La discusión ambiental ha sido reducida a una cuestión técnica. Se considera a la naturaleza como externa al ser humano (FOLADORI, S/F, p. 134).

Con respecto a estas aseveraciones que hacen hincapié en las diversas problemáticas, en donde se señala que estas están teniendo repercusiones en otros aspectos de la vida, como la salud, principalmente, en la pobreza, en el agotamiento de los recursos (el agua) y en donde se menciona que el hombre es el responsable al no hacer consciencia, esta entonces, sería la razón por la que las universidades tomaran en cuenta que se debe hacer algo, y respecto al currículo, la medida sería la incorporación del elemento medio ambiente.El papel de las universidades mexicanas para formar los profesionistas que puedan impulsar un desarrollo ambientalizado se reconoce en 
el Plan de Acción para el Desarrollo Sustentable en las Instituciones de Educación Superior (ANUIES, 2001). Sin embargo algunas universidades, no lo consideran así. Una investigación en la (BUAP), sobre la identificación del eje medio ambiente en el currículo, destacó que los participantes atribuyen los problemas ambientales principalmente a causas culturales -inconsciencia, falta de educación e irresponsabilidad-; seguidas de causas económicas -sobreexplotación-; políticas -gobierno corrupto-; tecnológicas -fábricas y autos-; sobrepoblación y urbanización; sociales y naturales. El paso por la universidad influye poco en la representación que tienen los alumnos sobre las causas de los problemas ambientales. La actual crisis ambiental puede explicarse por razones políticas, sociales, económicas, culturales y éticas (LARA, et al. 2010, p. 40-55).En el campo educativo, cuando se elabora un currículo, es necesario desarrollar el proceso de la contextualización, es en esta parte donde se identifican las problemáticas y con ello se vinculan las necesidades a atender en él. Una problemática es lo que sucede en el medio ambiente, esto se vuelve una necesidad atender en materia educativa. La contextualización curricular constituye un proceso a través del cual, las propuestas curriculares se ajustan a los parámetros particulares de los diversos entornos, instituciones y colectivos donde se aplicarán. En dicho proceso, la lógica de lo general, lo estandarizado, lo prescriptivo se contrapone a la lógica de lo local, lo situacional, lo adaptativo. Se trata de una visión de la educación y de la función de las escuelas contraria a la homogeneización y respetuosa con la diversidad (ZABALZA, 2012, p. 6).

\section{MATERIALES Y MÉTODOS}

La investigación es de campo, la información se obtuvoa través de la entrevista y la encuesta. La entrevista se aplicó a directivos, la encuesta se realizó con alumnos y profesores de tres unidades académicas (se describen en la muestra) de la Universidad Autónoma de Guerrero en la Ciudad de Acapulco. Se elaboró una rúbrica para analizar si figura el eje medio ambiente en los planes de estudio de las tres escuelas. Este estudio es bibliográfico, ya que se analizaron algunos documentos publicados. Es unainvestigación mixta, los análisis y los procedimientos, toman en cuenta la explicación y la descripción.Los diseños mixtos representan el más alto grado de combinación o integración entre los enfoques cualitativo y cuantitativo (HERNÀNDEZ et al, 2003: PEREIRA, 2011: BEDOLLA et al., 2015: p. 1-11). 


\section{Población}

Tabla I. Datos generales de la Universidad Autónoma de Guerrero en relación a número de profesores y estudiantes.

\begin{tabular}{cccc}
\hline \multicolumn{3}{c}{${ }^{*}$ Universidad Autónoma de Guerrero (UAGro), Guerrero, México(Profesores de nivel licenciatura) } \\
\hline Docentes & Mujeres & Hombres & Total \\
\hline & 383 & 779 & 1162 \\
\hline \multicolumn{4}{c}{${ }^{* *}$ Universidad Autónoma de Guerrero (estudiantes de nivel medio superior y superior) } \\
\hline Estudiantes & Mujeres & Hombres & Total \\
\hline & 43,468 & 39,302 & 83,770 \\
\hline
\end{tabular}

Fuente: *Anuario Estadístico 2013-2014 de la Universidad Autónoma de Guerrero/**Anuario Estadístico 2014-2015 de la Universidad Autónoma de Guerrero

\section{Muestra}

Tabla II. Datos específicos de las instituciones seleccionadas para obtener información con la encuesta.

\begin{tabular}{lcc}
\hline & $\begin{array}{c}\text { Universidad Autónoma de Guerrero (UAGro). } \\
\text { (Profesores y estudiantes en las unidades académicas seleccionadas). }\end{array}$ \\
\hline Unidad Académica o Facultad & $\begin{array}{c}\text { Total } \\
\text { docentes }\end{array}$ & Muestra seleccionada \\
\hline Facultad Sociología (Acapulco, México). & 15 & $\mathbf{4}$ \\
\hline Facultad Economía (Acapulco, México). & 15 & $\mathbf{1 1}$ \\
\hline Facultad Derecho (Acapulco, México). & 48 & $\mathbf{4 4}$ \\
\hline Total & 78 & $\mathbf{5 9}$ \\
\hline Universidad Autónoma de Guerrero, (UAGro) & (Estudiantes de nivel licenciatura) & \\
\hline Unidad Académica o Facultad & Total estudiantes & Muestra seleccionada \\
\hline Facultad Sociología (Acapulco, México). & $\mathbf{2 8 7}$ & 105 \\
\hline Facultad Economía (Acapulco, México). & 259 & 91 \\
\hline Facultad Derecho (Acapulco, México). & 2044 & 318 \\
\hline Total & 2590 & $\mathbf{5 1 4}$ \\
\hline
\end{tabular}

Fuente:Anuario Estadístico 2014-2015 de la Universidad Autónoma de Guerrero

*Cabe mencionar, que se aplicó una entrevista, esta fue dirigida a personal directivo, una al Director de la Unidad Academia de Sociología, otra al Subdirector de la Unidad Académica de Ciencias Sociales (Derecho) y otra al Subdirector de la Unidad Academia de Economía, con ello se dice, que se necesitó de otros tres elementos más en la muestra seleccionada. Estas personas se consideraron informantes claves sobre el currículo.

Técnicas empleadas para la recolección de información:

- Sellevó a cabo una entrevista con el personal directivo de las unidades académicas elegidas con el propósito de identificar el eje ambiental en el currículo, también se revisó en el modelo educativo dicho eje y aunado a ello se consiguieron los tres currículos de los programas que imparten la escuela de sociología, de economía y de derecho para realizar un análisis sobre la presencia del elemento ambiente.

- Se diseñó y aplicó una encuesta con las variables (percepción del problema, currículo y competencias) a estudiantesy maestros. El propósito de este instrumento fue comprender si el elemento medio ambiente está incorporado en los planes de estudio.La aplicación de esta técnica 
comprendió del 6 de marzo al 15 de octubre de 2015 y fue procesada a través del programa IBM SPSS Statistics 20. Posteriormente, se llevó a cabo el análisis para deliberar si el elemento medio ambiente está o no considerado. Los resultados se describen a través de un análisis respectivo.

\section{RESULTADO}

Con relación a las técnicas empleadas y a los objetivos de este estudio, se presentan los siguientes resultados:

Se realizó una entrevista al personal directivo de la Unidad Académica de Sociología (Director), de Derecho (Subdirector) y de Economía (Subdirector), facultades dependientes de la Universidad Autónoma de Guerrero, ubicada en Acapulco, Guerrero, México. Estas se desarrollaron durante el periodo marzo a octubre de 2015, el objetivo siempre fue llegar a lo que se quería conocer "El elemento medio ambiente está incorporado en estos planes de estudios", planteado como una hipótesis en cualquier momento.

Tabla III.Resultado de la entrevista realizada al personal directivo de las unidades académicas de Sociología, Derecho y Economía.

\begin{tabular}{llll}
\hline \multicolumn{2}{l}{ Unidades Académicas de la Universidad Autónoma de Guerrero } & Sociología \\
\hline Pregunta & Respuesta & $\begin{array}{l}\text { Economía } \\
\text { Respuesta }\end{array}$ & $\begin{array}{l}\text { Derecho } \\
\text { Respuesta }\end{array}$ \\
\hline $\begin{array}{l}\text { ¿El currículo de la } \\
\begin{array}{l}\text { Institución tiene integrado } \\
\text { el elemento medio ambiente? }\end{array}\end{array}$ & $\begin{array}{l}\text { No, definitivamente, no. } \\
\text { Porque no tenemos mate- } \\
\text { rias que se relacionen con } \\
\text { el medio ambiente. }\end{array}$ & $\begin{array}{l}\text { Sí, porque hay unas } \\
\text { materias que si hablan del } \\
\text { medio ambiente. }\end{array}$ & $\begin{array}{l}\text { Creo que si porque hay unas materias } \\
\text { que llevan medio ambiente. }\end{array}$ \\
\hline
\end{tabular}

El Modelo Educativo de la UAG 2004 con reformas en el 2013 de la Universidad Autónoma de Guerrero como se cita en las primeras líneas de este documento, muestra la necesidad de incorporar el elemento medio ambiente como método transversal para coadyuvar en la sustentabilidad social. Con respecto a ello, se procedió a realizar un análisis en los planes de estudios que contemplan estas tres instituciones. Teniendo como fundamento lo que estipula dicho modelo educativo, para hacer este análisis se tuvo que diseñar una rúbrica con las variables (elementos del plan de estudio), como lo son fundamentos del plan de estudio, finalidad y perfiles del plan de estudio, selección y organización de las competencias y programas de unidades de aprendizaje, en estos cuatro elemento debía de identificarse la presencia del elemento medio ambiente, para lo cual, se evaluó con los parámetros (muy vinculado, parcialmente vinculado, poco vinculado, no se vincula). Los resultados mostraron que los tres planes de estudios van de poco vinculado a no se vinculan). Esto da a comprender que los tres planes de estudio no están contemplando la inserción del elemento medio ambiente como se estipula 
en el documento rector de la UAGro. Cabe mencionar, que el programa de Derecho y Economía integra una o dos materias que se relacionan con el medio ambiente, pero no es suficiente. El modelo lo indica como un método transversal y como lo expresan las teorías citadas, con enfoque hacia el desarrollo sustentable.

La encuesta se procesó cuantitativamente y su análisis fue cualitativo, esta considero las variables (percepción del problema, currículo y competencias), la encuesta fue aplicada a la muestra seleccionada como se muestra en la tabla II del presente y fue para estudiantes y maestros. Para medir las variablesse consideraron los elementos, “Deficiente”, “Apenas Aceptable”, "Bien”, "Muy Bien", "Excelente" y "No Aplica”, que fueron opciones para responder en el instrumento aplicado, estos mismos se tomaron en cuenta para medir cualitativamente, el nivel de desarrollo en relación a las variables de estudio, por decir, la percepción del problema ambiental, la incorporación del elemento medio ambiente en el currículo y las competencias ambientales con que cuenta el docente y las que están desarrollando los estudiantes. Se presentan los resultados a continuación:

Tabla IV.Resultado de la encuesta (profesores).

\begin{tabular}{lcccc}
\hline Universidad Autónoma de Guerrero, México & & \\
\hline Variables & $\begin{array}{c}\text { Facultad de } \\
\text { Sociología }\end{array}$ & $\begin{array}{c}\text { Facultad de } \\
\text { Economía }\end{array}$ & $\begin{array}{c}\text { Facultad de } \\
\text { Derecho }\end{array}$ & Observación \\
\hline $\begin{array}{l}\text { Percepción del } \\
\text { problema }\end{array}$ & Deficiente & $\begin{array}{c}\text { Apenas } \\
\text { Aceptable }\end{array}$ & $\begin{array}{c}\text { Apenas } \\
\text { Aceptable }\end{array}$ & Apenas comprenden la problemática ambiental. \\
\hline $\begin{array}{l}\text { Currículo } \\
\text { Apenas } \\
\text { ambientales } \\
\text { de los } \\
\text { docentes }\end{array}$ & $\begin{array}{c}\text { Apenas } \\
\text { Aceptable }\end{array}$ & Deficiente & $\begin{array}{c}\text { Apenas } \\
\text { Aceptable }\end{array}$ & Apenas contemplan (EA) en el currículo. \\
\hline
\end{tabular}

Tabla V. Resultado de la encuesta (estudiantes).

\begin{tabular}{ccccc}
\hline & \multicolumn{2}{c}{ Universidad Autónoma de Guerrero, México } \\
\hline Variables & $\begin{array}{c}\text { Facultad de } \\
\text { Sociología }\end{array}$ & $\begin{array}{c}\text { Facultad de } \\
\text { Economía }\end{array}$ & $\begin{array}{c}\text { Facultad de } \\
\text { Derecho }\end{array}$ & Observación \\
\hline $\begin{array}{c}\text { Percepción del } \\
\text { problema }\end{array}$ & Deficiente & Bien & $\begin{array}{c}\text { Apenas } \\
\text { Aceptable }\end{array}$ & Apenas conciben el problema ambiental. \\
\hline Currículo & Deficiente & Bien & $\begin{array}{c}\text { Apenas } \\
\text { Aceptable }\end{array}$ & $\begin{array}{c}\text { Apenas contempla el currículo el } \\
\text { elemento medio ambiente y por ende la (EA). }\end{array}$ \\
\hline $\begin{array}{c}\text { Competencias } \\
\text { en los alumnos }\end{array}$ & $\begin{array}{c}\text { Apenas } \\
\text { Aceptable }\end{array}$ & Bien & Deficiente & Apenas desarrollan competencias ambientales. \\
\hline
\end{tabular}




\section{DISCUSIÓN Y CONCLUSIONES}

Todo lo que tiene vida en este mundo persiste por razones de la dependencia a los recursos que la naturaleza ofrece, si estos se terminan, se corre el riesgo de exterminar la existencia. Según, (FOLADORI, S/Fb p.84), el ser humano no puede hacer nada cuyo producto o resultado no sea, asimismo, natural, ya que él es, en sí, naturaleza. En el caso del hombre, este, es parte de la naturaleza, no debemos separarlos, sin embargo, se concibe como el responsable principal de los daños que se están causando. Los perjuiciosocasionados a la naturaleza repercuten en el medio. Actualmente el concepto "ambiente" engloba la multicausalidaddelosprocesosfísicos,sociales, económicos, tecnológicos y biológicos, al igual que la complicada red de interrelaciones y los múltiples niveles espacio-temporales en las que éstas se dan. Con este criterio, Enrique Leff nos señala que "naturaleza" representa una categoría (Pacheco, 2005, p. 30). Con esta aserción, se comprende entonces que la incorporación del elemento ambiental en el currículo no debe atender solamente temas que se relacionen con "naturaleza", como la ecología, por ejemplo. Lo que se pretende al implementar dicho elemento es, desarrollar procesos que interrelacionen, medio ambiente, naturaleza, sociedad, economía, cultura, entre otros. Como lo establece (MACEDO, et al. 2007, b) compactible con el desarrollo sostenible. Los resultados obtenidos en esta investigación, denotan que las instituciones seleccionadas apenas incorporan el elemento ambiental, una definitivamente no. Las dos que si lo implementan, solo presentan de una a dos disciplinas que se relacionan con ello, sin embargo, el modelo educativo de la universidad, no lo considera así, esta lo especifica como método transversal. La educación ambiental (EA) en México, si bien tuvo un inicio tardío respecto a otros países, norteamericanos y europeos, ha mantenido un ritmo progresivo y creciente, sobre todo durante los últimos quince años (GONZÀLEZ, 2000, p.3). La Universidad en cuestión contempla el elemento medio ambiente desde el año 2004 en su modelo educativo y últimamente, por consiguiente, porque no aparece como tal en las currículos de las escuelas analizadas, acaso cuando se elaboraron los planes de estudio, no se consideró lo estipulado en dicho documento. En el caso de la Unidad Académica de Sociología, este tuvo una reforma curricular en el 2009 y 2012 y aun así es la que no tiene una asignatura que se relaciona con medio ambiente. Con respecto al desarrollo de competencias para implementar por parte de los docentes estrategias de educación ambiental en el proceso de aprendizaje, no se cuenta del todo con ellas, si bien, los profesores de economía y de derecho que imparten una asignatura apegada a la cuestión ambiental, que no son muchos. En el caso de los estudiantes, la mayoría solo está formando competencias en el área de su formación, pero en lo que respecta a las ambientales, estas no son 
consideradas y los que sí, es por el hecho de que toman una unidad de aprendizaje que se relaciona con el elemento ambiental, que igual que los maestros, son muy pocos.

Para concluir este apartado, es necesario que la Universidad Autónoma de Guerrero, realice un diagnóstico para identificar los programas educativos que se han actualizado curricularmente y que han considerado lo que plantea el modelo educativo en materia de implementar el elemento ambiental, en el caso de que existan deficiencias, es necesario tomar las medidas necesarias, como por ejemplo informar de acerca su incorporación. Aunado a ello, los docentes responsables del currículo en su unidad académica deben estar al tanto del tema en cuestión y atender las recomendaciones del modelo educativo. Para todos los profesores, también, es necesario llevar cursos de actualización para formar competencias y estas sirvan para desarrollar estrategias didáctico-pedagógicas en materia ambiental en el proceso de aprendizaje. Las competencias que formen los estudiantes tienen que tener implícitas las ambientales, con el fin de que contribuyan a la sustentabilidad social que el mundo requiere.

\section{REFERENCIAS}

BEDOLLA, S. R. et al.Evaluación del Desempeño acorde a las Competencias Docentes del nivel superior en la UAGro. Unidad Académica de Enfermería, Foro de Estudios sobre Guerrero, n. 1, p. 1-11.Nov. 2015.

BRAVO, W. Estudio de los conocimientos de los profesores sobre Educación Ambiental. Didáctica Ambiental, n. 9, p. 58, mar. 2011.

CHAGOLLÀN, F. A. et al. Educación Ambiental. 1. Ed. México: UMBRAL, 2006.

GONZÀLEZ, G. E. La Educación Ambiental en México: Logros, perspectivas y retos de cara al nuevo milenio. Congreso III Congreso Iberoamericano de Educación Ambiental. MÈXICO, p.3.

GUIER, S. E. et al. Educación Ambiental en Costa Rica. Tendencias evolutivas, perspectivas y desafíos. v. 18, n. (1-2), p. 4, 2004. Disponible en: file:///C:/Users/MiPc/ Downloads/EA_Tendencias_UNED_2004\%20(1).pdf Acceso en: 22 abr. 2016.

FOLADORI, G. (S/F). Una tipología del pensamiento ambientalista. Capítulo 3. P. 134. Disponible en: http:// estudiosdeldesarrollo.net/coleccion_america_latina/ sustentabilidad/Sustentabilidad6.pdf Acceso en: 19 abr. 2016.

HOPWOOD, B. et al. Sustainable Development: Mapping Different Approaches..v. 13, p. 39. 
HONDURAS, Guía para transversalizar el eje ambiental en las carreras del nivel de educación superior de Honduras. República de Honduras, 2009. p. 6. Disponible en: https://redambientalinteruniversitaria.files.wordpress. com/2016/o1/guia-para-transverzalizar-el-eje-ambientalen-las-carreras-de-educacio-superior-en-honduras.pdf Acceso en: 22 abr. 2016.

LARA, G. J; et al. Representación social de las causas de los problemas ambientales. El caso de la Benemérita Universidad Autónoma de Puebla. Trayectorias Trayectorias, v. 12, n. 30. p. 40-55. Disponible en: http:// www.redalyc.org/pdf/607/60713488004.pdf Acceso en: 22 abr. 2016.

MACEDO, B. et al. Educación ambiental y educación para el desarrollo sostenible en América Latín.v. 1, p. 33. 2007. Disponible en: http://www.ehu.eus/cdsea/web/revista/ numero_1/o1_03macedo.pdfAcceso en: 19 abr. 2016.

MICILIO, C. LOS PROBLEMAS AMBIENTALES, ¿SON DE APTITUD O DE ACTITUD? v. 17, p. 69-74. 2009. Disponible en: http://www.ambientum.com/revista/2009/mayo/ problemas.asp Acceso en: 19 abr. 2016.

PROGRAMA DE LAS NACIONES UNIDAS PARA EL MEDIO AMBIENTE (PNUMA). GEO 5(2012). Perspectiva del medio ambiente mundial. Resumen para responsables de políticas, 2012. p. 6-7.

PACHECO, M. M. El ambiente. Más allá de la naturaleza. Elementos, v. 57, p. 30, p.30. 2005. Disponible en:http:// garritz.com/andoni_garritz_ruiz/documentos/Lecturas. CS.\%20Garritz/Pensamiento.Ambientalista/Ambiente. Elementos.57.Pacheco.pdfAcceso en: 19 abr. 2016.

SALVADOR, S. M. ¿Es importante la transversalidad ambiental en los planes curriculares?. Delos, España, v. 12, n. 3, p. 1, feb. 2013. Disponible en: http://www.eumed.net/ rev/delos/16/transversalidad-ambiental.html Acceso en: 19 abr. 2016.

UAG. Modelo Educativo y Académico, 2004. Chilpancingo, Gro. México. p. 46-47.

UAGro. Modelo Educativo Hacia una educación de calidad con inclusión social, 2013. Chilpancingo, Gro. México. pp. 62-63.

UAGro. Anuario Estadístico 2013-2014 de la Universidad Autónoma de Guerrero.

UAGro. Anuario Estadístico 2014-2015 de la Universidad Autónoma de Guerrero. p. 47-49. 
29 ANÁLISIS DEL CURRÍCULO Y OTROS ACTORES DEL PROCESO EDUCATIVO PARA IDENTIFICAR EL EJE AMBIENTAL: INFORME PROYECTO

ZAMBRANO, J, G. et al. Tendencias Modernas y Postmodernas dela Educación Ambiental. Sapiens, Caracas, v. 11, n. 1. pp. 202-203. 2010. Disponible en: http://www2. scielo.org.ve/scielo.php?script $=$ sci_arttext\&pid $=\mathrm{S} 1317-$ $58152010000100012 \& \operatorname{lng}=\mathrm{es} \& \mathrm{nrm}=\overline{\mathrm{i}}$

ZAVALZA, B. M. Territorio, cultura y contextualización curricular. Interaccoes, v. 8, n. 22, p. 6. Disponible en: http://revistas.rcaap.pt/interaccoes/article/view/1534/ 1225Acceso en: 19 abr. 2016.

Recebido em: 04/11/2016 Aprovado em: 30/11/2016 